\title{
Computation of outflow rates from accretion disks around black holes
}

\author{
S. Das, I. Chattopadhyay, A. Nandi, and S. K. Chakrabarti \\ S. N. Bose National Centre for Basic Sciences, Salt Lake, Kolkata 700098, India
}

Received 15 May 2001 / Accepted 30 August 2001

\begin{abstract}
We self-consistently estimate the outflow rate from the accretion rates of an accretion disk around a black hole in which both the Keplerian and the sub-Keplerian matter flows simultaneously. While Keplerian matter supplies soft-photons, hot sub-Keplerian matter supplies thermal electrons. The temperature of the hot electrons is decided by the degree of inverse Comptonization of the soft photons. If we consider only thermallydriven flows from the centrifugal pressure-supported boundary layer around a black hole, we find that when the thermal electrons are cooled down, either because of the absence of the boundary layer (low compression ratio), or when the surface of the boundary layer is formed very far away, the outflow rate is negligible. For an intermediate size of this boundary layer the outflow rate is maximal. Since the temperature of the thermal electrons also decides the spectral state of a black hole, we predict that the outflow rate should be directly related to the spectral state.
\end{abstract}

Key words. X-rays: stars - stars: winds, outflows - black hole physics

\section{Introduction}

Most of the galactic black hole candidates are known to undergo spectral state transitions (Tanaka \& Lewin 1995; Chakrabarti \& Titarchuk 1995, hereafter CT95; Ebisawa et al. 1996). Two common states are the so-called hard state and the soft state. In the former, soft-X-ray luminosity is low and the energy spectral index $\alpha \sim 0.5$ $\left(E_{\nu} \propto \nu^{-\alpha}\right)$ in the $2-10 \mathrm{keV}$ range. In the latter state, the soft-X-ray luminosity is very high, and hard-X-ray intensity is negligible. There is also a weak power-law hardtail component with an energy spectral slope $\alpha \sim 1.5$. In the two component advective flow (TCAF) model (CT95), the viscous Keplerian disk resides in the equatorial plane, while the weakly viscous sub-Keplerian flow flanks the Keplerian component both above and below the equatorial plane. The two components merge into a single component when the Keplerian disk also become sub-Keplerian. It is suggested (Chakrabarti 1990) that close to a black hole, at around $10-15 r_{\mathrm{g}},\left(r_{\mathrm{g}}=2 G M_{\mathrm{BH}} / c^{2}\right.$ is the Schwarzschild radius, $M_{\mathrm{BH}}$ and $c$ are the mass of the black hole and the velocity of light respectively) the sub-Keplerian flow slows down due to the centrifugal barrier and becomes hotter. Chakrabarti (1999, hereafter Paper I) shows that this centrifugal pressure-supported boundary layer (CENBOL for

\footnotetext{
Send offprint requests to: S. K. Chakrabarti,

e-mail: chakraba@boson.bose.res.in

* Honorary Scientist, Centre for Space Physics, 114/v/1A Raja S.C. Mullick Rd., Kolkata 700047.
}

short) region could be responsible for the generation of thermally-driven outflowing winds and jets and computed the ratio of the outflow to the inflow rate assuming a simple conical accretion disk model.

In the present paper, we compute the absolute value of the outflow rate as a function of the rates of the two inflow components, Keplerian and sub-Keplerian. This we do analytically following the recently developed procedure of obtaining shock locations (Das et al. 2001). By dynamically mixing these two components using solutions of the viscous transonic flows we obtain the specific energy and angular momentum of the sub-Keplerian region. We use these pair of parameters to locate shocks in the flow, compute the compression ratio and from this, the outflow rate. We note that as Keplerian matter is increased in the mixture, the shock compression ratio goes down, and the outflow rate decreases. This is also the case even from a radiative transfer point of view - when the Keplerian rate is high, the CENBOL region is completely cooled and the shock compression ratio $R \sim 1$. Hence in the soft state, which is due to increase of the Keplerian rate, outflow should be negligible.

In the next section, we present the governing equations to compute the outflow rates using a purely analytical method. We compute results for both the isothermal and adiabatic outflows. In Sect. 3, we present our results for a single component sub-Keplerian flow. We also produce examples of realistic disks with Keplerian and sub-Keplerian components and obtain outflow rates as functions of the 
inflow parameters. In Sect. 4, we discuss our results and draw conclusions.

\section{Model equations}

We consider matter accreting on the equatorial plane of a Schwarzschild black hole. Spacetime around the black hole is described by the Paczyński-Wiita pseudo-Newtonian potential $\phi=\frac{G M_{\mathrm{BH}}}{r-2 G M_{\mathrm{BH}} / c^{2}}$ (Paczyński \& Wiita 1980) where $M_{\mathrm{BH}}$ is the mass of the black hole and $G, c$ are the gravitational constant and velocity of light respectively. Here, $r$ is the radial distance from the origin of the coordinate in which the black hole is treated at the centre. We use geometric units in which all the length, time and velocity scales are measured in units of $2 G M_{\mathrm{BH}} / c^{2}$, $2 G M_{\mathrm{BH}} / c^{3}$ and $c$ respectively. In future, we use $r$ to denote non-dimensional distance, $\vartheta$ and $a$ to denote the nondimensional radial velocity and adiabatic speed of sound respectively. In accretion or outflow, we assume that the viscous stress is negligible so that matter moves with a constant specific angular momentum. Indeed, even if viscosity is not negligible, the transport of angular momentum is slow compared to the infall timescale. Hence, matter can have almost constant specific angular momentum.

In this case, the radial momentum equation for a non-dissipative flow in vertical equilibrium is given by (Chakrabarti 1989),

$\vartheta \frac{\mathrm{d} \vartheta}{\mathrm{d} r}+\frac{1}{\rho} \frac{\mathrm{d} P}{\mathrm{~d} r}-\frac{\lambda^{2}}{r^{3}}+\frac{1}{2(r-1)^{2}}=0$.

Integrating this, we obtain the conserved specific energy of the flow,

$\mathcal{E}_{v}=\frac{1}{2} \vartheta^{2}+n a^{2}+\frac{\lambda^{2}}{2 r^{2}}-\frac{1}{2(r-1)}$,

where $n$ is the polytropic index of the inflow and $\lambda$ is the specific angular momentum. In Eq. (1), $P$ and $\rho$ are thermal pressure and density respectively, $v$ is the infall velocity and $a=\sqrt{(} \gamma P / \rho)$ is the adiabatic sound speed.

The mass flux conservation equation in a flow which is in vertical equilibrium is given by,

$\dot{M}_{\text {in }}=4 \pi \rho \vartheta r h(r)=\Theta_{\text {in }} \rho_{\mathrm{s}} \vartheta_{\mathrm{s}} r_{\mathrm{s}}^{2}$,

where $\Theta_{\text {in }}\left(=\sqrt{\frac{2 n}{n+1}} 4 \pi a_{\mathrm{s}} r_{\mathrm{s}}^{1 / 2}\right)$ is the solid angle subtended by the inflow at the CENBOL boundary. Subscripts "s" denote the quantities at shock (CENBOL boundary) and $h(r)=\sqrt{\frac{2}{\gamma}} a r^{1 / 2}(r-1)$ is the half-thickness of the disk in vertical equilibrium at a radial distance $r$.

A sub-Keplerian flow with a positive energy will pass through the outer sonic point and depending on whether the Rankine-Hugoniot condition is satisfied or not, a standing shock may form (Chakrabarti 1990; Chakrabarti 1996). If a standing shock forms, then the post-shock flow would become hotter and would emit hard X-ray radiation. This CENBOL region behaves similarly to the boundary of a normal star; it would be expected to drive outflows. Using Eqs. (2) and (3), it is easy to obtain shock locations (i.e., outer surface of the CENBOL) analytically. Briefly, the procedure to obtain shocks involves the following steps:

(a) For a given pair of specific energy $\mathcal{E}_{v}$ and angular momentum $\lambda$, one obtains a quartic equation for the sonic point and solves it for the three sonic points located outside the horizon. Two of them are saddle type or "X" type sonic points and one is a centre type or "O" type sonic point.

(b) From the inner and the outer "X" type points, Mach numbers are expressed as polynomials of radial distance $r$. These Mach number expressions satisfy constraints that they must have appropriate values at the sonic points.

(c) In addition, it is enforced that the Mach number invariants at the shock location are also satisfied $\left(r_{\mathrm{s}}\right)$.

(d) The resulting equation becomes quartic in $r_{\mathrm{s}}$ and the shock locations are obtained from its solution.

Details are discussed in Das et al. (2001). We consider only the region of the inflow parameter space $\left(\mathcal{E}_{v}, \lambda\right)$ that is able to produce standing shocks.

In the pre-shock region, matter is cooler and is subKeplerian. Assuming $\mathcal{E}_{v} \sim 0$ (freely falling condition) and $a \sim 0$ (cool gas) in presence of angular momentum, matter will fall with a velocity,

$\vartheta(r)=\left[\frac{1}{r-1}-\frac{\lambda^{2}}{r^{2}}\right]^{1 / 2}$.

Using this, from Eq. (3) the density distribution can be obtained.

At the shock $r=r_{\text {s }}$, i.e., the boundary of the CENBOL, the compression ratio is given by,

$R=\frac{\Sigma_{+}}{\Sigma_{-}}=\frac{h_{+}\left(r_{\mathrm{s}}\right) \rho_{+}\left(r_{\mathrm{s}}\right)}{h_{-}\left(r_{\mathrm{s}}\right) \rho_{-}\left(r_{\mathrm{s}}\right)}=\frac{\vartheta_{-}}{\vartheta_{+}}$,

where subscripts "-" and "+" refer, respectively, to quantities before and after the shock. Here, $\Sigma$ is the density of matter integrated vertically $(\Sigma \sim \rho h)$ and the second "=" sign was written using the mass flux conservation equation given above (Eq. (3)).

At the shock, the total pressure (thermal and ram pressure) is balanced:

$W_{-}\left(r_{\mathrm{s}}\right)+\Sigma_{-}\left(r_{\mathrm{s}}\right) \vartheta_{-}^{2}\left(r_{\mathrm{s}}\right)=W_{+}\left(r_{\mathrm{s}}\right)+\Sigma_{+}\left(r_{\mathrm{s}}\right) \vartheta_{+}^{2}\left(r_{\mathrm{s}}\right)$,

where $W$ is the pressure of the gas integrated vertically.

We assume that in the pre-shock region, the thermal pressure is small in comparison to the ram pressure,

$W_{+}\left(r_{\mathrm{s}}\right)=\frac{R-1}{R} \Sigma_{-}\left(r_{\mathrm{s}}\right) \vartheta_{-}^{2}\left(r_{\mathrm{s}}\right)$.

The isothermal sound speed in the post-shock region is obtained from:

$C_{\mathrm{s}}^{2}=\frac{W_{+}}{\Sigma_{+}}=\frac{R-1}{R^{2}} \vartheta_{-}^{2}=\frac{1}{f_{0}}\left[\frac{r_{\mathrm{s}}^{2}-\lambda^{2}\left(r_{\mathrm{s}}-1\right)}{r_{\mathrm{s}}^{2}\left(r_{\mathrm{s}}-1\right)}\right]$,

where, $f_{0}=\frac{R^{2}}{R-1}$. 
Up to the sonic point matter moves slowly and the density is higher. Since the outflow would take place in a sea of radiation, the momentum deposition is likely to be effective. With the electron number density $n_{\mathrm{e}} \propto r^{-3 / 2}$, yet photon number density $n_{\gamma} \propto r^{-2}$, it is easier to deposit momentum only close to the black hole. In radiation driven outflows from the stellar surface, it is customary to assume flows to be isothermal until the sonic point. We first compute outflow rates making this assumption. Later we drop this assumption and show that the general behaviour remains similar. In addition, we assume that there is very little rotation in the outflow. There is no a priori reason to assume this, except that there is no observational support of rotation in the jet and it is possible that due to radiative viscosity most of the angular momentum is transported very close to the black hole. Furthermore, it has been observed that the effect of angular momentum in the outflow is to bring the sonic points closer to the black hole, especially away from the axis (Sakurai 1985; Chakrabarti 1986). The general effect would produce a transverse structure in the jet which we ignore in the present solution. It was shown (Das \& Chakrabarti 1999) that in presence of angular motion the conical outflow is to be replaced by an annular flow confined by the centrifugal barrier and the funnel wall. Generally speaking, the outflow surface varies as $r^{3 / 2}$. However, the inflow surface area is still proportional to $r^{2}$. Because of this asymmetry, the problem is no longer tractable analytically and is beyond the scope of the present paper.

\subsection{When the outflow is isothermal}

The radial momentum balance equation in the outflow is given by

$\vartheta \frac{\mathrm{d} \vartheta}{\mathrm{d} r}+\frac{1}{\rho} \frac{\mathrm{d} P}{\mathrm{~d} r}+\frac{1}{2(r-1)^{2}}=0$,

and the continuity equation is given by

$\frac{1}{r^{2}} \frac{\mathrm{d}}{\mathrm{d} r}\left(\rho \vartheta r^{2}\right)=0$.

From above equations we get

$\frac{\mathrm{d} \vartheta}{\mathrm{d} r}=\frac{N}{D}$

where

$N=\frac{2 C_{\mathrm{s}}^{2}}{r}-\frac{1}{2(r-1)^{2}} \quad$ and $\quad D=\vartheta-\frac{C_{\mathrm{s}}^{2}}{\vartheta}$.

To obtain the sonic point condition, we put $N=0$ and $D=0$ and get, $\vartheta\left(r_{\mathrm{c}}\right)=C_{\mathrm{s}}$, and $r_{\mathrm{c}}=\frac{1+8 C_{\mathrm{s}}^{2} \pm \sqrt{1+16 C_{\mathrm{s}}^{2}}}{8 C^{2}}$, where the subscript $\mathrm{c}$ denotes the quantities at the sonic point in the outflow.

Integrating the radial momentum equation, considering the sonic point condition, we have,

$C_{\mathrm{s}}^{2} \ln \rho_{+}-\frac{1}{2\left(r_{\mathrm{s}}-1\right)}=\frac{1}{2} C_{\mathrm{s}}^{2}+C_{\mathrm{s}}^{2} \ln \rho_{c}-\frac{1}{2\left(r_{\mathrm{c}}-1\right)}$.
Here, we have ignored the radial velocity in the outflow at the boundary of the shock. Using the notations $\rho\left(r_{\mathrm{c}}\right)=\rho_{\mathrm{c}}$ and $\rho\left(r_{\mathrm{s}}\right)=\rho_{+}$, we obtain,

$\rho_{\mathrm{c}}=\rho_{+} \exp [-f]$

where $f=\frac{1}{2}-\frac{1}{2 C_{\mathrm{s}}^{2}} \frac{r_{\mathrm{s}}-r_{\mathrm{c}}}{\left(r_{\mathrm{s}}-1\right)\left(r_{\mathrm{c}}-1\right)}$.

The outflow rate is given by

$\dot{M}_{\text {out }}=\Theta_{\text {out }} \rho_{\mathrm{c}} \vartheta_{\mathrm{c}} r_{\mathrm{c}}^{2}$,

where $\Theta_{\text {out }}$ is the solid angle subtended by the outflow.

From Eqs. (2) and (15) we get,

$$
\begin{aligned}
\frac{\dot{M}_{\text {out }}}{\dot{M}_{\text {in }}}= & R_{\dot{m}}=\frac{\Theta_{\text {out }}}{\Theta_{\text {in }}}\left[\frac{r_{\mathrm{s}}^{2}\left(r_{\mathrm{s}}-1\right)}{r_{\mathrm{s}}^{2}-\lambda^{2}\left(r_{\mathrm{s}}-1\right)}\right]^{-1 / 2} \frac{R C_{\mathrm{s}} r_{\mathrm{c}}^{2}}{r_{\mathrm{s}}\left(r_{\mathrm{s}}-1\right)} \\
& \times \exp [-f] .
\end{aligned}
$$

The above relation is very similar to that obtained in Paper I when the effects of rotation in the inflow were ignored. However, there the ratio $R_{\dot{m}}$ was a function of $R$ alone. In the present analysis, $R$ is computed selfconsistently from the specific energy and the specific angular momentum of the flow:

$R=\frac{\Sigma_{+}}{\Sigma_{-}}=\frac{\vartheta_{-}}{\vartheta_{+}}=\left[\frac{\frac{1}{2} M_{+}^{2}+n}{\frac{1}{2} M_{-}^{2}+n}\right]^{1 / 2}$,

where pre-shock and post-shock Mach numbers $M_{-}(\mathcal{E}, \lambda)$ and $M_{+}(\mathcal{E}, \lambda)$ are computed analytically from Das et al. (2001).

\subsection{When the outflow is adiabatic}

At the other extreme, when the energy of the outflow does not change, one can also obtain an analytical expression for the outflow rate assuming the $r_{\mathrm{s}}>>\lambda^{2}$. In this case, the entropy density of the flow in the post-shock region is the same at the entropy density of the entire outflow and the specific energy is also conserved along the outflow. We assume that the turbulence generated at the CENBOL has effectively transported angular momentum away. Thus, the energy conservation equation gives

$n a_{\mathrm{s}}^{2}-\frac{1}{2 r_{\mathrm{s}}}=\frac{2 n+1}{2} a_{\mathrm{c}}^{2}-\frac{1}{2 r_{\mathrm{c}}}$,

where the left hand side is the energy at the CENBOL $\left(r=r_{\mathrm{s}}\right)$ and the right hand side is at the sonic point $\left(r=r_{\mathrm{c}}\right)$ of the outflow where $u_{\mathrm{c}}=a_{\mathrm{c}}$ has been used. $n=\frac{1}{\gamma-1}$ is the polytropic constant. In a Bondi (in or out) flow, $a_{\mathrm{c}}^{2}=1 / 4 r_{\mathrm{c}}$. At the CENBOL, $a_{\mathrm{s}}^{2}=\gamma C_{\mathrm{s}}^{2}$, where $C_{\mathrm{s}}$ is the isothermal sound speed (Eq. (8)). Using these, one obtains (assuming $r_{\mathrm{s}}>>\lambda^{2}$ )

$\frac{r_{\mathrm{c}}}{r_{\mathrm{s}}}=\frac{2 n-3}{4\left(\frac{2 n \gamma}{f_{0}}-1\right)}$,

and

$\frac{a_{\mathrm{c}}^{2}}{a_{\mathrm{s}}^{2}}=\frac{f_{0} r_{\mathrm{s}}}{4 \gamma r_{\mathrm{c}}}$. 


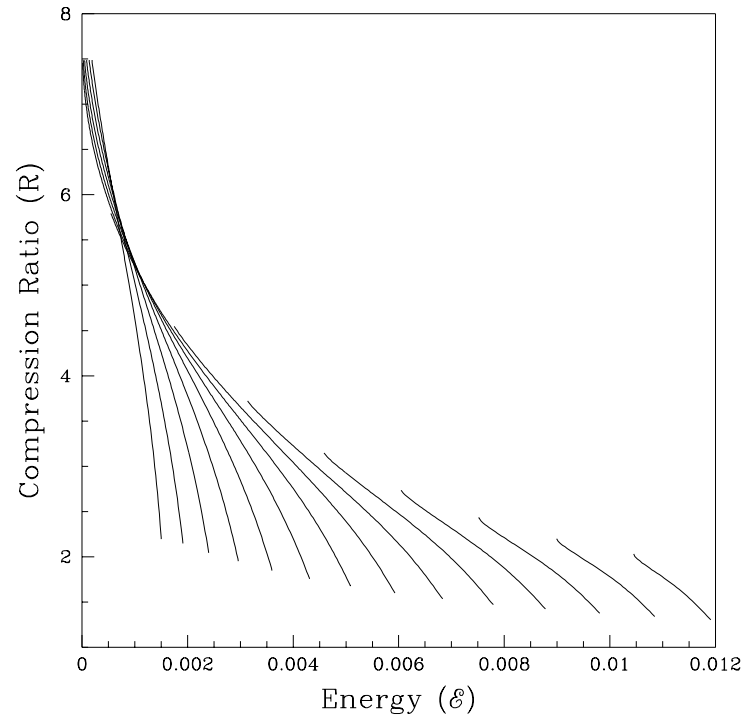

Fig. 1. Variation of the compression ratio of the shocks as a function of specific energy $\mathcal{E}$ and angular momentum $\lambda$ as obtained from the analytical solution. $\lambda$ varies from 1.57 (right) to 1.79 (left). Curves are drawn at intervals of $\mathrm{d} \lambda=0.02$.

In an adiabatic flow with an equation of state $P=K \rho^{\gamma}$ (where $K$ is a constant and a measure of entropy), one obtains, assuming, $K_{\mathrm{c}}=K_{\mathrm{s}}$,

$\frac{\rho_{\mathrm{c}}}{\rho_{\mathrm{s}}}=\left(\frac{a_{\mathrm{c}}^{2}}{a_{\mathrm{s}}^{2}}\right)^{n}$.

From these relations one obtains the ratio of the outflow to the inflow rate as

$R_{\dot{m}}=\frac{\Theta_{\mathrm{o}}}{\Theta_{\mathrm{i}}}\left(\frac{f_{0}}{4 \gamma}\right)^{3} \frac{R}{2}\left\{\frac{4}{3}\left[\frac{8(R-1)}{R^{2}}-1\right]\right\}^{3 / 2}$.

Here, we have used $n=3$ for a relativistic flow. The nature of this function will be discussed below.

\section{Outflow rates from inflow parameters}

In Eq. (16), we presented the outflow/inflow rate ratio as a function of the compression ratio of the flow at the shock. The compression ratio is obtained from the specific energy and angular momentum using Eq. (17). First, we employ analytical means to obtain this for a single component sub-Keplerian disk. Second, we use a two component Keplerian/sub-Keplerian disk to actually compute these parameters from more fundamental parameters such as accretion rates and viscosity.

\subsection{Single component sub-Keplerian flows}

In Fig. 1, we plot the analytical solution of the compression ratio $R$ as a function of the flow parameters: specific energy $\mathcal{E}$ and the specific angular momentum $\lambda$. The shock strength generally increases when energy decreases and the angular momentum increases. This is because for low energy, the outer sonic point and the shock form very

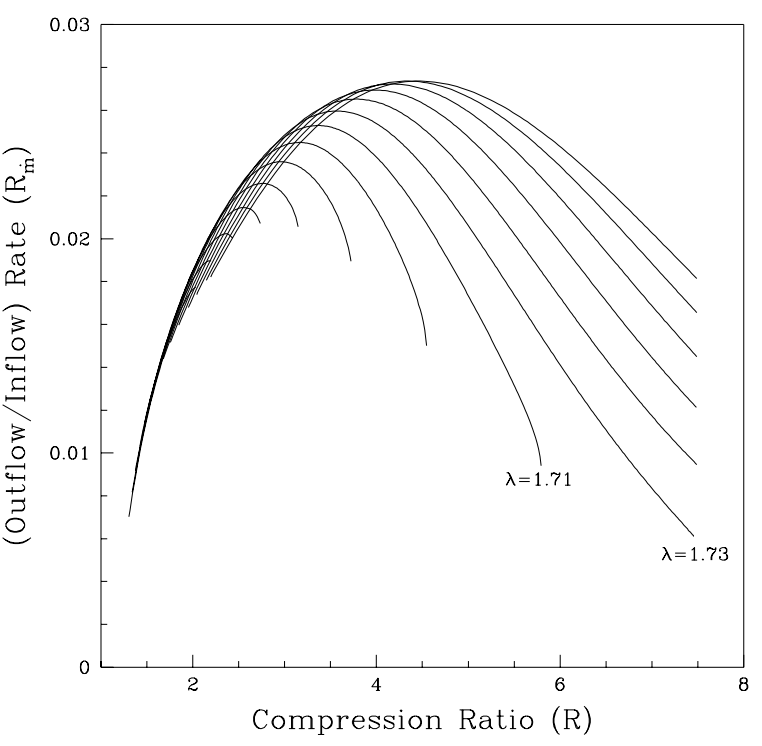

(a)

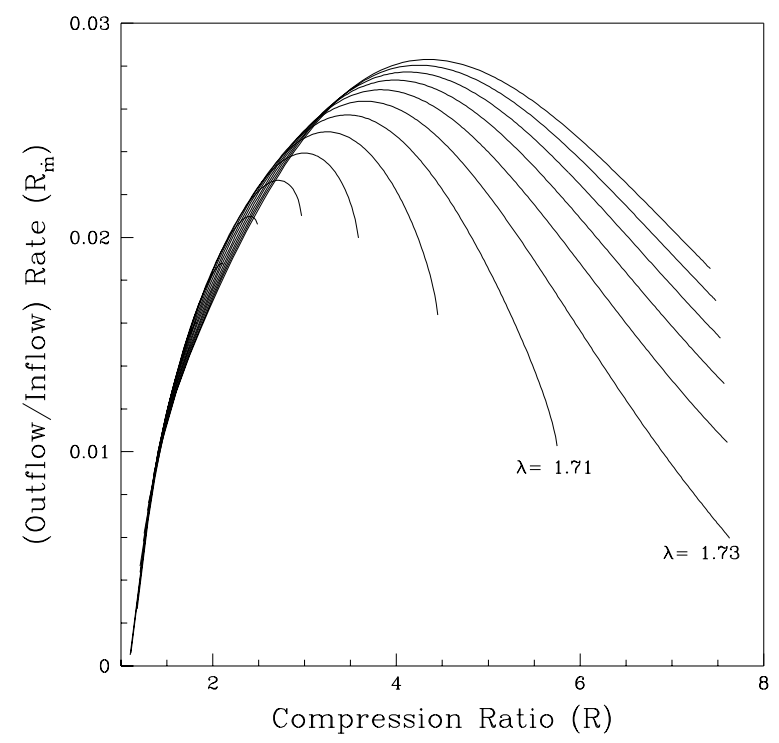

(b)

Fig. 2. a) Variation of the ratio of outflow to inflow rates $R_{\dot{m}}$ as a function of compression ratio for various specific angular momenta. $\lambda=1.57$ (inner most) to 1.83 (outer most). Curves are drawn at intervals of $\mathrm{d} \lambda=0.02$. Outflow rate is maximum at some intermediate compression ratio; b) Same as Fig. 2a except that curves are drawn for the exact numerical solution.

far away and the Mach number jumps from a very large number to a very small number. If the angular momentum is decreased, shock is produced only if the specific energy is high, i.e., if the sonic points and the shocks are very close to the black hole. Here, flow becomes subsonic before its Mach number could be very high.

Figure 2a shows the principle result of our work when only one sub-Keplerian accretion is chosen as the inflow. We plot the ratio $R_{\dot{m}}$ for a large number of specific angular momenta of the flow ranging from 1.57 (innermost) to 1.83 (outermost) at intervals of $\mathrm{d} \lambda=0.02$. The curves are 
drawn for all ranges of specific energy $\mathcal{E}$ for which shocks are formed. Along the $X$-axis the compression ratio $R$ of these shocks is written. Here to compute solid angles of the inflow and the outflow, we assume the half opening angle of the outflow to be $10^{\circ}$. Therefore, $\Theta_{\text {out }}=\pi^{3} / 162$. $\Theta_{\text {in }}$ is given in the discussion following Eq. (2). In Paper I, the compression ratio $R$ was assumed to be a parameter and no angular momentum was assumed a priori. Presently, we show the dependence on angular momentum. The general character, namely, that the outflow rate is negligible when the shock is weak $(R \sim 1)$ and falls off gradually for strongest shock $(R \rightarrow 7)$, remains the same as in Paper I, however. There is a peak at about $R_{\dot{m}} \sim 2.8 \%$. Note that for a given $R, R_{\dot{m}}$ increases monotonically with specific angular momentum $\lambda$. This is because density of CENBOL rises with $\lambda$. The curves corresponding to $\lambda=1.71$ and 1.73 are specially marked since there is a clear difference in tendency of the variation of $R_{\dot{m}}$. For instance, below $\lambda \sim 1.72$, very strong shocks are not possible at all and as a result the outflow rate has a lower limit. For $\lambda \gtrsim 1.72$ such a limit does not exist.

The general behaviour of the outflow rate can be understood in the following way: when shocks are strong, they form very far out, and thus, even though the CENBOL area (which is basically the area of the base of the jet) increases, the net outflow rate is low. When the shock forms very close to the black hole, the temperature is high, and thus the outflow velocity is larger, but the CENBOL surface area goes down. Thus the product is low. For the intermediate cases the net effect is larger.

For comparison with the analytical work presented in Fig. 2a, in Fig. 2b we present a similar diagram drawn using a numerical computation of the shock locations (Chakrabarti 1989). Excellent agreement between these two figures implies that the approximations on which the analytical work was based are justified. All the features are reproduced well in Fig. 2a, except that for the weakest shocks outflow rate is not as low as in the numerical calculation of Fig. $2 \mathrm{~b}$.

We now present the nature of $R_{\dot{m}}$ when the outflow is also chosen to be adiabatic in Fig. 3. We used $\Theta_{\mathrm{o}} / \Theta_{\mathrm{i}} \sim 0.1$ for reference. We observe that the peak is still located at around $R=\sim 4$ and the outflow rate drops for very strong $(R \sim 7)$ and very weak $(R \sim 1)$ shocks. We therefore believe that our conclusion about the behaviour of $R_{\dot{m}}$ is generic.

\subsection{Two component advective flows}

Chakrabarti \& Titarchuk (1995) proposed that the spectral properties are better understood if the disk solutions of sub-Keplerian flows are included along with the Keplerian flows. Recently, Smith et al. (2001a), Smith et al. (2001b), Miller et al. (2001) found conclusive evidence of these two components in many of the black hole candidate accretion flows. While the matter with higher viscosity flows along the equatorial plane as a Keplerian

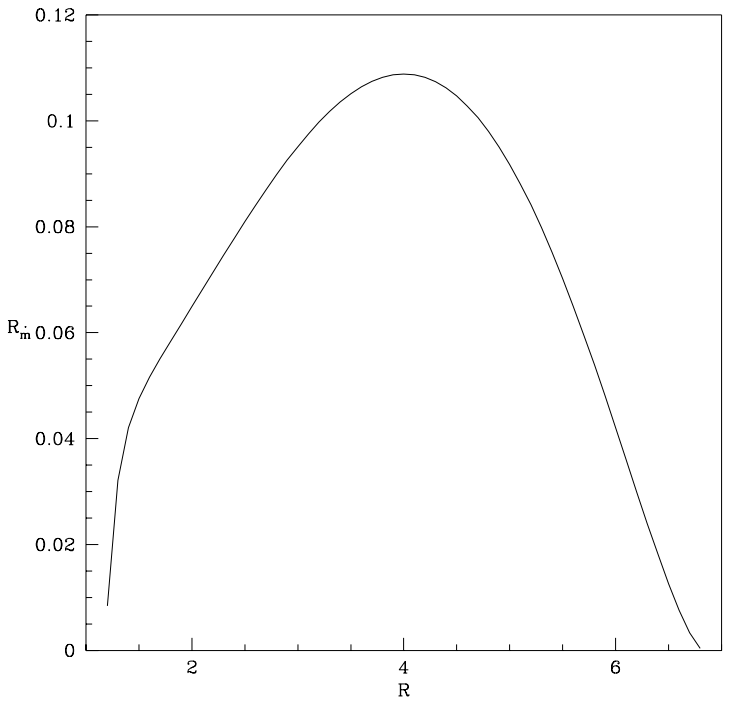

Fig. 3. Ratio of the outflow and the inflow rates as a function of the compression ratio of the inflow when the outflow is adiabatic. The general nature of the function remains the same as that of the isothermal outflow.

disk (of rate $\dot{M}_{\mathrm{K}}$ ), sub-Keplerian halo matter (of rate $\dot{M}_{\mathrm{h}}$ ) with lower viscosity flanks the Keplerian disk above and below (Fig. 4a). Since the inner boundary condition on the horizon forces the flow to be sub-Keplerian, irrespective of their origin (Chakrabarti 1990, 1996) matter mixes (at say, $r=r_{\text {tr }}$ ) from both the Keplerian and sub-Keplerian flows before entering a black hole to form a single component sub-Keplerian with an average energy and angular momentum of $\mathcal{E}$ and $\lambda$ respectively. The specific energy and angular momentum of the mixed flow is computed from:

$\mathcal{E}=\frac{\dot{M}_{\mathrm{K}} \mathcal{E}_{\mathrm{K}}+\dot{M}_{\mathrm{h}} \mathcal{E}_{\mathrm{h}}}{\dot{M}_{\mathrm{K}}+\dot{M}_{\mathrm{h}}}$

and

$\lambda=\frac{\dot{M}_{\mathrm{K}} \lambda_{\mathrm{K}}+\dot{M}_{\mathrm{h}} \lambda_{\mathrm{h}}}{\dot{M}_{\mathrm{K}}+\dot{M}_{\mathrm{h}}}$.

Here, $\mathcal{E}_{\mathrm{K}}, \mathcal{E}_{\mathrm{h}}, \lambda_{\mathrm{K}}$ and $\lambda_{\mathrm{h}}$ are the specific energies and specific angular momentum of the Keplerian and the subKeplerian components at $r=r_{\text {tr }}$ respectively.

Figure 4a shows a schematic diagram of the crosssection of a two-component accretion flow. The transition radius $\left(r=r_{\text {tr }}\right)$ where the Keplerian disk becomes sub-Keplerian, and the shock location $r=r_{\mathrm{s}}$, are indicated. Figure 4b shows two solutions (marked I and II) of the equations governing a two-component flow (Chakrabarti 1996) where $\lambda_{\mathrm{d}} / \lambda_{\mathrm{K}}$ (Sub-Keplerian matter from the Keplerian disk) and $\lambda_{\mathrm{h}} / \lambda_{\mathrm{K}}$ (Sub-Keplerian halo) are plotted as a function of the logarithmic radial distance. Viscosities chosen for these two components are $\alpha=0.04$ and $\alpha=0.01$ respectively. For $r<r_{\text {tr }}=45$ (lightly shaded region) the two sub-Keplerian flows mix to create a single component. For simplicity, we assume viscosity to be negligible in this region. Thus, the specific angular momentum and specific energy computed at 


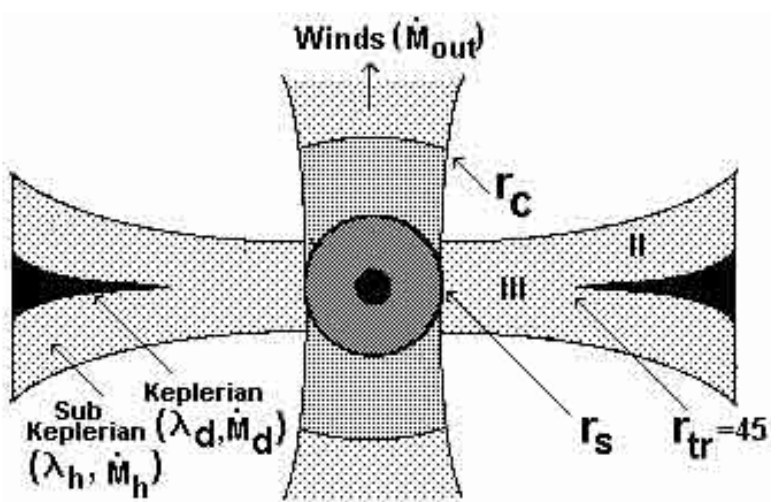

(a)

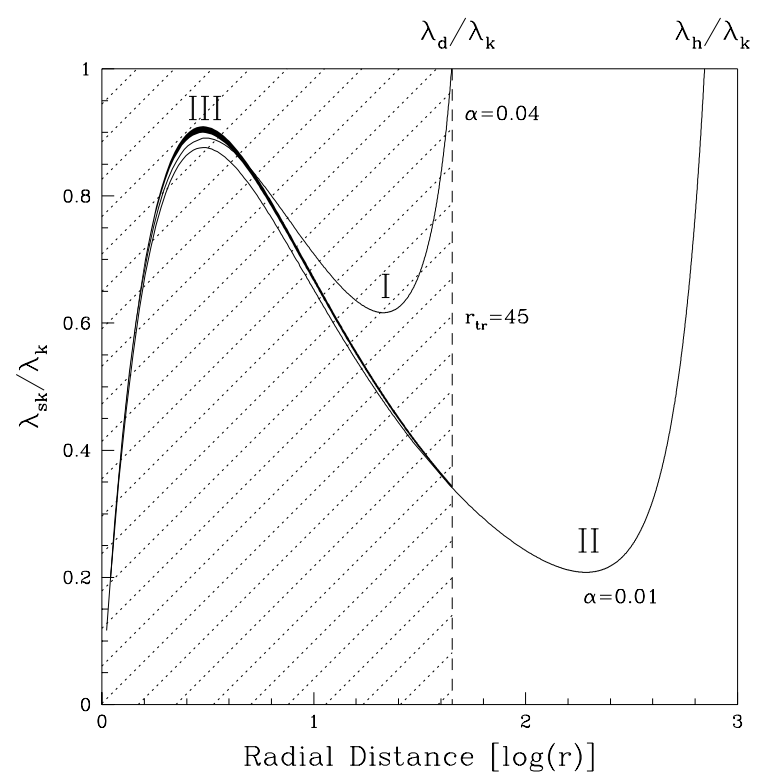

(b)

Fig. 4. a) Schematic diagram of the cross section of twocomponent accretion flow. See text for details; b) Solution of the two-component flow equations for two different viscosities. They are merged to form a single solution as depicted in Fig. 4a.

$r=r_{\text {tr }}$ from Eqs. (22) and (23) remain constant $(\lambda)$ for $r<r_{\mathrm{tr}}$. Dark solid curve (marked III) shows the angular momentum distribution $\lambda / \lambda_{\mathrm{K}}$ of all possible mixtures of the two components which allow shock formation. We chose a case where $\dot{M}_{\mathrm{d}}+\dot{M}_{\mathrm{h}}=2.0 \dot{M}_{\text {Edd }}$ and vary the Keplerian component $\dot{M}_{\mathrm{d}}$ where $\dot{M}_{\text {Edd }}$ is the Eddington accretion rate.

In Fig. 5, the computed outflow rates are shown when the half opening angle of the outflow is $10^{\circ}$. In this case, $\frac{\Theta_{\text {out }}}{\Theta_{\text {in }}} \sim \sqrt{\frac{n+1}{2 n}} \frac{\pi^{2}}{648 a_{\mathrm{s}} r_{\mathrm{s}}^{1 / 2}}$. The left axis shows the rate of outflow $\dot{m}_{\text {out }}=\dot{M}_{\text {out }} / \dot{M}_{\text {Edd }}$ as a function of the Keplerian disk rate (right panel) $\left(\dot{m}_{\mathrm{d}}=\dot{M}_{\mathrm{d}} / \dot{M}_{\mathrm{Edd}}\right)$ and the halo rate (upper panel) $\left(\dot{m}_{\mathrm{h}}=\dot{M}_{\mathrm{h}} / \dot{M}_{\text {Edd }}\right)$. The lower axis gives the compression ratio at the shock. The most important conclusion that can be drawn here is that the
Accretion Rate of Halo $\left(\dot{m}_{h}\right)$

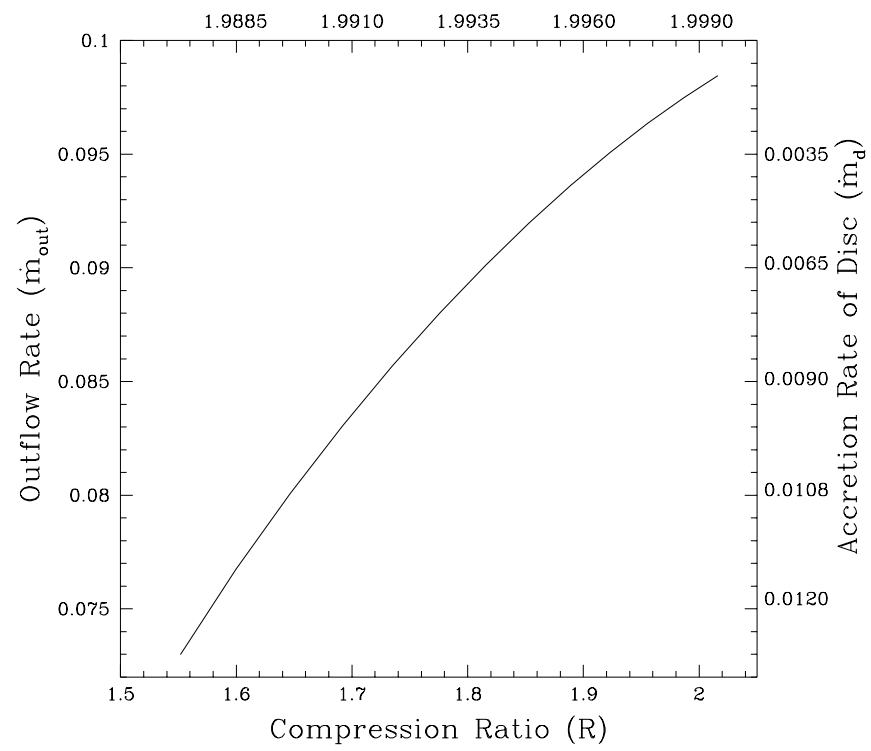

Fig. 5. Variation of outflow rates (left axis) with compression ratio at shocks (lower axis). The upper axis gives the variation of sub-Keplerian accretion rate and right axis gives the same for Keplerian accretion rate.

outflow rate steadily goes up as the Keplerian disk rate $\dot{m}_{\mathrm{d}}$ decreases and the spectrum goes to a harder state. When the Keplerian rate is higher, the compression ratio is lower and the outflow rate is also lower. This conclusion, drawn completely from dynamical considerations, is also found to be true from the spectral studies (CT95) where it was shown that the post-shock region cools down and the shock disappears $(R \rightarrow 1)$. Our work therefore hints that the outflow would be negligible in softer states.

\section{Discussion and concluding remarks}

CT95 pointed out that the centrifugal pressure-supported boundary layer (CENBOL) of a black hole accretion flow is responsible for the spectral properties of a black hole candidate. In this Paper, we present analytical results to show that this CENBOL is also responsible for the production of the outflows, and the outflow rate is strongly dependent on the inflow parameters, such as specific energy and angular momentum. We showed that in general, the outflow rate is negligible when the shock is absent and very small when the shock is very strong. In intermediate strength, the outflow rate is the highest. As the specific angular momentum is increased, the outflow rate is also increased. This conclusion is valid when the flow is either isothermal or adiabatic.

We also demonstrated how a realistic two-component flow (TCAF) consisting of Keplerian and sub-Keplerian components produces a significant amount of outflow. Since matter close to a black hole is sub-Keplerian by nature, the two components must mix to form a single sub-Keplerian flow which has positive specific energy and almost constant specific angular momentum. We showed 
that as the Keplerian rate of the disk is increased, the outflow rate is decreased as the shock compression ratio approaches unity. This conclusion, drawn from a dynamical point of view, is also corroborated by the spectral behavior as well - as the Keplerian rate is raised, the postshock region is cooled due to inverse Comptonization and the shock disappears. This reduces the thermal pressure drive and the resulting outflow rate is reduced.

Acknowledgements. This work is partly supported by a project (Grant No. SP/S2/K-14/98) funded by Department of Science and Technology (DST), Govt. of India.

\section{References}

Chakrabarti, S. K. 1986, ApJ, 303, 582

Chakrabarti, S. K. 1989, ApJ, 347, 365 (C89)

Chakrabarti, S. K. 1990, Theory of Transonic Astrophysical Flows (Singapore: World Sci.) (C90)
Chakrabarti, S. K., \& Titarchuk, L. G. 1995, ApJ, 455, 623

Chakrabarti, S. K. 1996, ApJ, 464, 664

Chakrabarti, S. K. 1999, A\&A, 351, 185 (Paper I)

Das, T. K., \& Chakrabarti, S. K. 1999, Class Quant. Grav, 16, 3879

Das, S., Chattopadhayay, I., \& Chakrabarti, S. K. 2001, ApJ, 557, 983

Ebisawa, K., Titarchuk, L. \& Chakarbarti, S. K. 1996, PASJ, 48, 59

Miller, J. M., et al. 2001, ApJ, submitted [astro-ph/0107514]

Paczyński, B., \& Wiita, P. J. 1980, A\&A, 88, 23

Sakurai, T. 1985, Astron. Astrophys., 152, 121

Smith, D. M., Heindl, W. A., \& Swank, J. H. 2001a, ApJ, submitted [astro-ph/0103304]

Smith, D. M., Heindl, W. A., Markwardt, C., \& Swank, J. H. 2001b, ApJ, 554, L41

Tanaka, Y., \& Lewin, W. H. G. 1985, in X-ray Binaries, ed. W. H. G. Lewin, J. van Paradijs, \& E. P. I. van den Heuvel (Cambridge University Press), 166 